\title{
Towards Patient-oriented Design: A Case of the Egyptian Private Outpatient Clinics
}

\author{
M. Heshmat \\ Egypt-Japan University of Science \\ and Technology, Alexandria, Egypt \\ mahmoud.heshmat@ejust.edu.eg \\ mheshmat@aun.edu.eg
}

\author{
N. Mostafa \\ Egypt-Japan University of Science \\ and Technology, Alexandria, Egypt \\ noha.ali@ejust.edu.eg
}

\author{
J. Park \\ Tokyo Institute of Technology, \\ Tokyo, Japan \\ park.j.ai@m.titech.ac.jp
}

\begin{abstract}
In this paper, outpatient clinics' systems are addressed and their common issues were discussed. As a case study, the private clinics in Egypt are used, and a designed service solution was synthesized. In the case study, the system and application was mainly physician-oriented rather than patient-oriented. With such physician-oriented systems alongside with the current design approaches, waiting times and appointment scheduling are two critical issues. Therefore, in this study, a design thinking approach was implemented to address these design problems for patients and clinic staffs. Based on that analysis, a design application solution called "YourClinic" was developed to support online booking and consultation. This study provides design features and functions to improve the quality of communication between patients and clinic staffs, reduce waiting time, and develop schedules for patients and staffs in order to move towards a patient-oriented clinic service.
\end{abstract}

\section{Introduction}

Recently, more attention was paid to provide reliable healthcare products, systems, and services to citizens; however, there are huge gaps between the developed and developing countries. The United States dedicated $17.9 \%$ of their Gross Domestic Product (GDP) to healthcare systems in 2012 [1], while Egypt dedicated only $4.7 \%[2,3]$. The developing countries have encountered challenges such as limitations on operating resources, high cost of medication, and inefficient management decision tools. In addition, demand on using outpatient clinics has been increasing due to the increase in population and life expectancy. People frequently visit outpatient clinics either for taking medical consultation and checkup or for receiving medication. Therefore, many researchers have addressed outpatient clinic research to identify some of the related problems. The most common problems are patients' waiting times, physician idle/over times, and patient congestion. Such problems were not addressed by the previous studies, but the operational analysis perspectives. For example, in [4], the authors used simulation to study the effect of different appointment schedules on an outpatient clinic. However, the results were friendlier with the service provider than customers. Laganga et al. [5] used simulation to test patient-scheduling rules with the objective to increase the service provider productivity. Berg et al. [6] developed booking strategies for the outpatient procedure centers, their objective was to maximize the expected profit. Wu et al. [7] stated that the most important performance measure in outpatient clinics should be the customers' waiting time not the revenue of the clinic. Cayirli and Veral [8] made a comprehensive survey about the outpatient scheduling, and concluded that the impact of the previous studies is still limited. Gupta and Denton [9] conducted a comprehensive survey on the appointment scheduling in health care and stated that mostly all the previous models ignore patient scheduling preferences [9]. Patients' preferences may include specific dates and times for consultation and also the selection of specific physicians according to some criteria. The success of any healthcare system depends strongly on the degree of patients' engagement with their service providers, and that is why designers have tried to understand the impact of care delivery and the effect of physical environment on human behavior to create valuable solutions [10]. Service providers differ greatly in their management of their time; some physicians add more capacity by double booking, working for extra hours to satisfy the increasing demand, whereas, others hold on firmly to their daily schedules. For instance, many physicians restrict the number of physical 
examinations everyday beforehand [9]. Nowadays, the competition in health care industry is increasing, and patients' profiles and requirements have great variability. Surveys indicate that patients select the health service providers according to their ability to respect their appointment times besides their medical proficiency; and accordingly, healthcare administrators cannot ignore the penalty of poorly designed appointment systems and focus only on the revenue [8]. Moreover, it is also arguable that physician's time is more important than that of the patients [8].

In this work, the following research questions are addressed:

1. Can the outcomes of the qualitative study on clinics provide an applicable solution?

2. What are the functions and features that would contribute in making outpatient clinics more patient-oriented?

To explore these two questions, a case project was conducted for the Egyptian private clinics. 'Time' was considered as the main issue, and a prototype is proposed to help patients to find an appropriate outpatient clinic, make an online appointment and also make online consultation if needed. Design thinking was invited as a problem-solving approach to identify problems inherited in the Egyptian outpatient clinics. The contributions of this paper can be summarized as follows:

1. Using a design thinking approach, we analyzed problems of the Egyptian private clinics' systems and explore its design opportunities.

2. Identifying latent communication issues between patients and clinic staffs.

3. Developing a mobile application as a design solution based on the design problems.

4. Taking a step toward a patient-oriented clinic from a physician-oriented one.

The remainder of the paper is organized as follows: section 2 gives a literature review, and then the methodology followed in this work is illustrated in section 3. In section 4, a case study with problem description is given for the situation of outpatient clinics in Egypt and also the related design issues. In section 5, an analysis of the problems that emerged from the brainstorming process is presented, then a solution for the problem is proposed in section 6 , finally, conclusions and future work are given in section 7 .

\section{Literature Review}

Singh and Chaudhary [11] compared different systems and software applications developed over time in healthcare, and concluded that all of these developed applications lack the applicability in developing countries due to the deficient of infrastructure and the high cost. Abd et al. [12] put a framework to improve clinical workflow process by integrating qualitative and quantitative tools, they also conducted a stakeholder analysis and a survey to improve the whole process. Almarri et al. [13] proposed criteria of how to select a suitable application in healthcare. Ong et al. [14] conducted a study that integrates smart phones as a self-management tool for patients in an outpatient clinic, and concluded that it is feasible and acceptable; similar study can be found in [15]. Chowdhary et al. [16] proposed a software architecture framework that can simplify using analytics in the mobile-healthcare applications.

Regarding using design thinking as a solution approach in healthcare, Brown et al. [17] reported that there is a fundamental challenge for designers to move from designing objects to designing socio-material assemblies. Therefore, there are few studies that apply the design thinking approach in healthcare systems. Carroll \& Richardson [18] concluded that it is desirable to establish a connected healthcare framework using design thinking principles. Hopkins et al. [19] investigated opportunities to build a followup platform using brainstorming and prototyping. Hsueh et al. [20] studied and implemented a platform support patient before, during, and after medical encounter. MacDonald and Niamat Elahee [21] argued that the design thinking can solve various issues which managers struggle with in crowd sourcing, and confirmed that design thinking is recommended to be used in online applications beside the existing offline versions. Watkins et al. [22] used a design thinking qualitative approach for healthcare services in Africa and applied the case study in Zambia. Cheung [23] applied the design thinking procedure to design a product to be used in operating rooms in Hong Kong. In the previous studies, researchers have investigated the opportunities of these systems and also conceptualized solution implications for offering new features and functions. Yet, it lacks the qualitative orientations for elucidating latent factors of human behaviors. Therefore, in this study, the design thinking approach was highlighted for characterizing a set of problems, dealing with users and users' information environments in creating a healthcare system design application in outpatient clinics. 


\section{Methodology}

Design thinking is used as a problem solving approach in business, information technology, education, and healthcare [24]. The term 'design thinking' has been part of the collective consciousness of design researchers since Rowe [25]. Dorst [26] investigated the design thinking to helps deal with complex problems between organizations and their design practices. Ambrose and Harris [25] put seven stages for a design thinking approach: 1) define, 2) research 3) ideate, 4) prototype, 5) select, 6) implement, and 7) feedback. Design thinking approach invited ethnographic techniques to grasp complex problems and deconstruct the issues in order to identify hidden or patients' unmet needs in the design research and analysis. The proposed application enables patient to select a clinic and the preferred date and time, and then the clinic staff can use the application to make their schedules based on the patients' reservations.

As a research methodology, we conducted a design thinking approach with the following manners. First, a brain storming session was conducted to identify the manifest and latent problems in the Egyptian outpatient private clinics. To address the problems, we conducted qualitative interviews with patients, nurses, and physicians. Second, we used Insight Matrix [28] to cluster the related set of problems. Third, a decision is made regarding problems-cluster selection, namely, problems which are related to information. This cluster was selected because it is relatively easier to solve and don't need big investments. Fourth, an application prototype was developed to improve the information system performance, and thus the system itself. This synthesized a mobile application prototype, focusing on new features and functions for patients, physicians, and nurses in the private clinics. This application might give good impact on patient, physician, and nurse. The patient can select the preferred clinic, then the preferred day and time. Accordingly, the clinic staff coordinates the work in the clinic and their schedules based on the selected time slots. Another function is the ability to make online consultation by inserting the medical complaints by a patient and receive some consultation by staff.

\section{Case Study}

As mentioned in the previous section that design thinking approach can create innovative healthcare solutions that are not only attractive but also efficient. The motivation behind choosing the Egyptian situation as a case study is that from experience, some general questions were present about the healthcare services in general and the outpatient clinics in particular inside Egypt: why are they not effective? The public opinion in addition to the media report that people in Egypt act and interact badly with healthcare facilities, but we think that the main reason behind that is that healthcare systems are actually poorly designed. In the next section, an overview is given about the current situation of the Egyptian healthcare system which suffers from the absence of using information systems. Another motivation is that most of the current design thinking solutions is tangible products rather than service quality especially in healthcare systems. Meanwhile, many healthcare design teams are now staffed with multidisciplinary people with different backgrounds in design, business, medicine, and social science to deal with the complexity of healthcare issues. The contribution of this paper is to design thinking to add value to healthcare systems especially outpatient clinics.

As a similar methodology, Azab et al. [29] made a prototype that deals with the container-trucking sector in Egypt using design thinking.

\subsection{The Current Situation in Egypt}

There are two types of outpatient clinics in Egypt: public outpatient clinics and private outpatient clinics. The public outpatient clinics are in the governmental hospitals, where patients pay a little amount of money to receive consultation, but the service quality is not qualified. On the other hand, the private outpatient clinics are more expensive, but the service quality is better than public. Private clinics are owned and managed by individual or group physicians assisted by nurses. Nurse in private clinics has two tasks: 1) making appointments and 2) assisting the physician in some minor activities. Nearly all the private clinics in Egypt open its doors afternoon because of another unique issue, that is most of the physicians work in governmental hospitals in the morning and go to their private clinics after that.

Private clinics in Egypt are totally physician-oriented clinics because the physician decides the clinic times according to his/her personal schedule. As a result, patient has no choice about choosing the day or the consultation time. Moreover, if a patient wants to see the physician, he/she has to go to the clinic, pay the consultation fees, and wait in the clinic until his/her turn. This is due to the absence of a proper appointment system. Few clinics accept booking by telephone. Therefore, the waiting time of patients inside the clinic is very long. Some patients may wait for several weeks to see a well-known or an experienced doctor! Furthermore, in most clinics, there is no database system to help the staff to record the 
history of a certain patient who goes to the clinic frequently. Figure 1 depicts the process diagram of patient flow in a typical private clinic in Egypt.

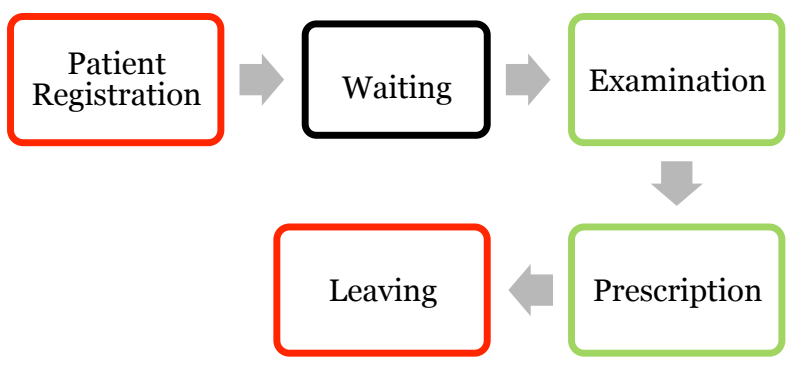

Figure 1. Process diagram for patient flow in private clinics

\subsection{Design Issues in Egyptian Private Clinics}

This section aims at presenting the design issues in private outpatient clinics in Egypt. After studying a typical private outpatient clinic in Egypt based on observation and interviews with patients, physicians, and nurses, and making physical simulation of the clinic environment and interaction between patients and clinic staff, a brain storming was conducted to deduct the problems found in the Egyptian private outpatient clinics as demonstrated in figure 2 .
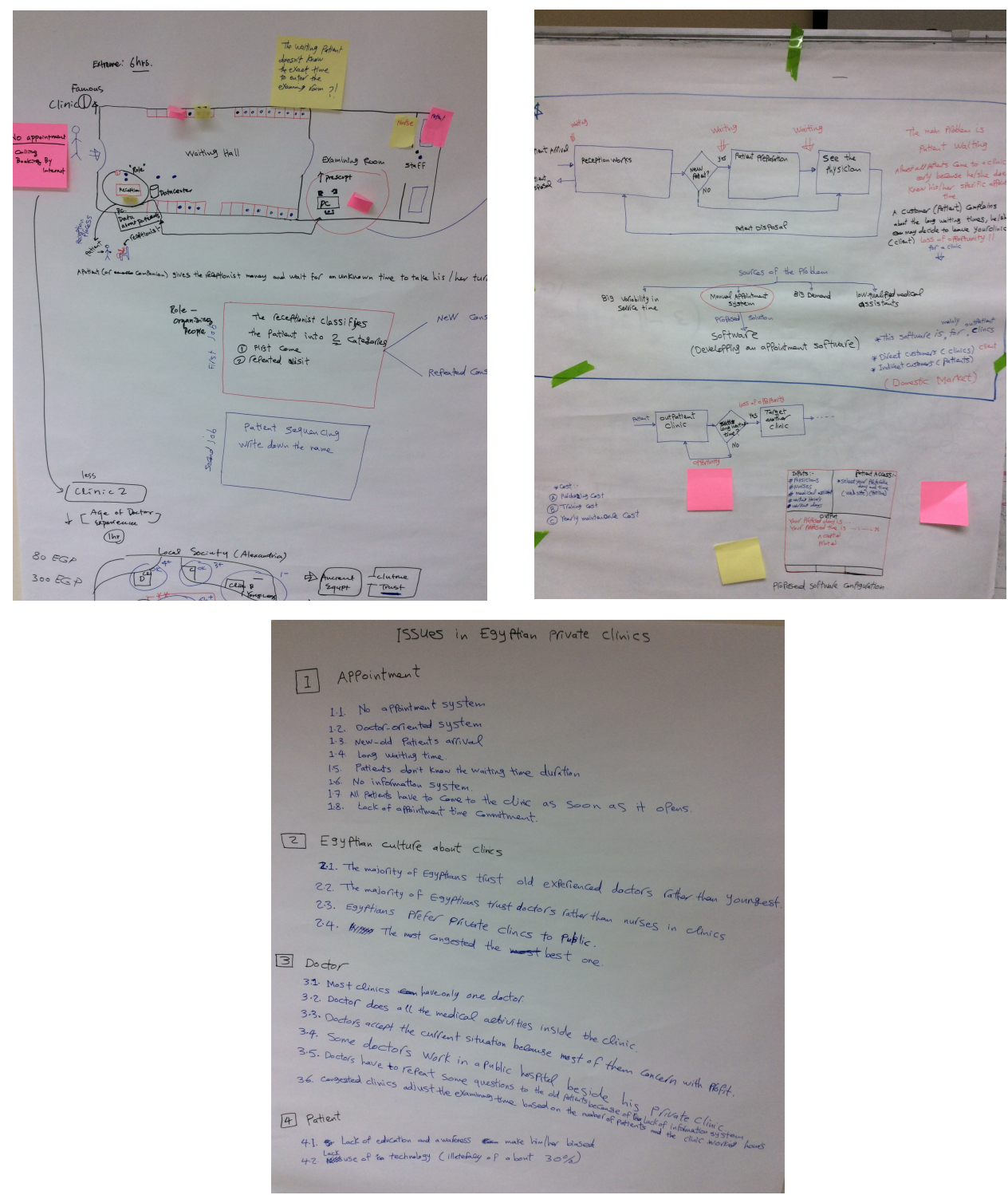

Figure 2. Snapshot of the brainstorming about private outpatient clinic issues in Egypt 
The brainstorming gave about twenty problems towards the overall problem of physician-oriented clinics. To give better understanding on the problem, Cause and effect diagram was developed (figure 3 ). It can be seen that there are six main causes for the problem; patients, process, system, staff, culture, and communication. Many of the patients who frequently visit outpatient clinics lack education and awareness on the consultation process and also commitment to the booking time he/she has held. The process itself contains issues such as the blind waiting times before the consultation due to the absence of time-slot scheduling, also congestion of patients inside clinics and unfair patient sequencing. The system as a cause has some feeders because of the absence of appointment system, ineffective information system to record patient's information for further consultations, and only physicians who own the clinic control everything in it. The fourth cause of the physician- oriented clinic problem is the staff. Physician does nearly all the medical activities inside the clinic and meanwhile focuses on profit other than the operation management of the whole clinic. Another issue regarding staff is that every clinic almost has a single physician even if there is congestion in the clinic. Culture plays a big role in the whole problem that is because most Egyptians think that the most congested clinic is the best one, therefore, they go to it which increases the problem. Furthermore, some of them believe that the older physician is better than the younger one. Another issue with the culture is that most of Egyptians do not trust nurses if they perform some medication activities inside the clinic which increase the burden upon physicians. The final cause of the physician-oriented problem is the lack of communication regarding follow up and online services.

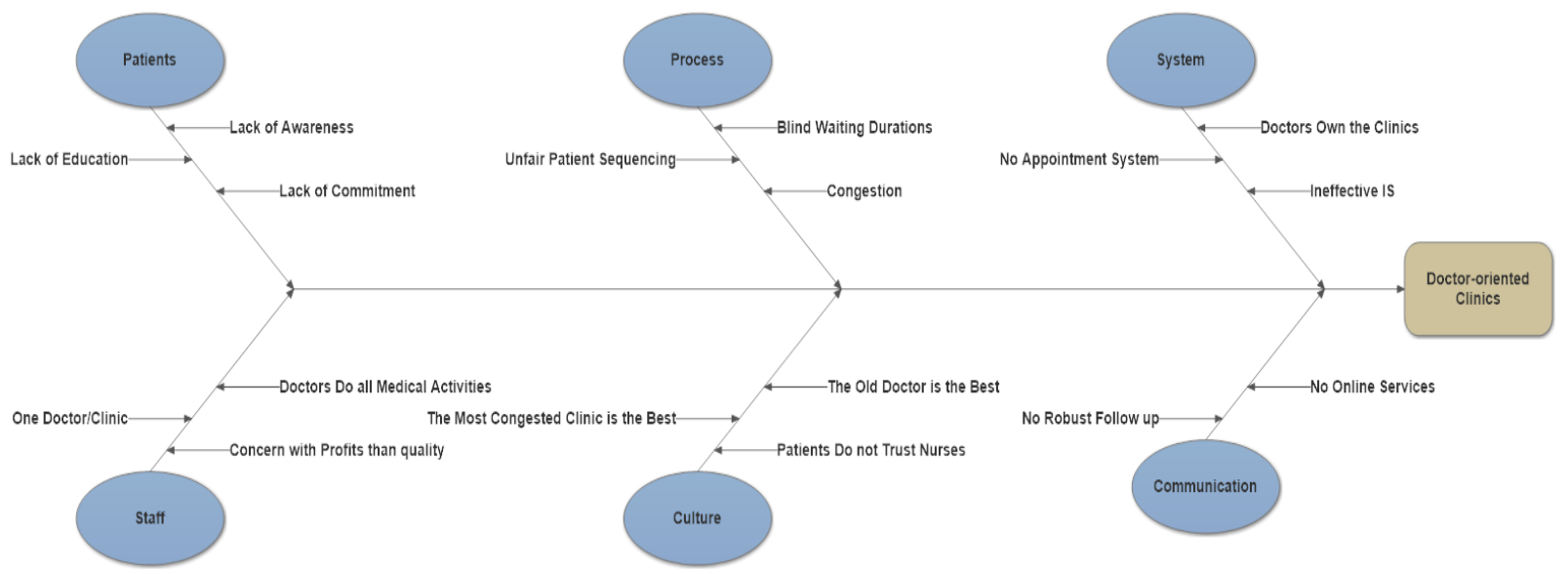

Figure 3. Cause and effect diagram for the Egyptian private outpatient clinics 


\section{Problem Analysis by using Insight Matrix}

Based on the design issues which are extracted from the Egyptian private outpatient clinics, twenty problems were identified to be analyzed using the Insight Matrix paradigm [28]. First, Insight Matrix was used to sort the twenty problems according to their impact on the whole problem. Second, it clustered every related group of problems in one cluster. The twenty problems are as follows:

1. The majority of Egyptians trust physicians than nurses in clinics.

2. The majority of Egyptians trust old and experienced physicians more than young physicians.

3. Most clinics have only one physician.

4. New-old patients' arrival ${ }^{1}$

5. Physician-based system

6. Doctors have to repeat some questions about their health to old patients although they were consulted before. ${ }^{2}$

7. Congested clinics adjust the examining time based on the number of waiting patients.

8. Physicians do all the medical activities inside the clinic.

9. Patients believe that the most congested is the best one.

10. Egyptians prefer private clinics to public.

11. Over-population

12. Lack of education and awareness

13. All patients have to come to the clinic as soon as it opens.

14. Long waiting time

15. No appointment system

16. Patients don't know the expected waiting duration.

17. No information system

\footnotetext{
${ }^{1}$ There are two types of patients arrive to outpatient clinics, new patients and old patients. Old patients are patients who have already visited the clinic before, and come to be checked again. The problem is that most doctors pay attention for new patients than old patients because new patients have to pay much more old ones. Therefore, old patients wait more than new ones.

${ }^{2}$ Because in most cases there is no system to store information about the patients to record and restore further. Every consultation time doctors deal old patients as new one and ask them the same question in previous consultation sessions.
}

18. Physicians accept the current situation because their focus is on profit.

19. Most of the physicians work in public hospitals beside their private clinics.

20. Lack of appointment time commitment

From the identified twenty problems, Insight Matrix paradigm was used (figure 4) to analyze them by sorting and clustering the most related problems in order to focus on the dominant problems.

The clustering process gives five clusters as shown in figure 5. It is obvious that the first four clusters need long-term solutions because they pertain with cultural and strategic reforms. So, the fifth cluster was chosen as the milestone problem of this research to develop a new application that tackles most of the problems in this cluster. The emerged problem tends to be about information. In other words, as there is no appointment technique that enables the patient to book his/her preferred time to go to a certain private clinic, the patient is forced to go to the clinic and make a registration according to the existing time slots and wait inside the clinic until his/her turn which is still unknown. Another source of the problem which is located in the information cluster is that nearly all doctors who have private clinics work in other places before the starting time of the clinic. As a result, the starting time of private clinics is not stationary but it is floating which increase the waiting burden upon patients. Some patients cannot stand the long waiting time and may abandon or can get outside the clinic for other matters and return again to the clinic which affects the commitment of patients with reservations and in the same time it is considered an opportunity loss for the clinic. 


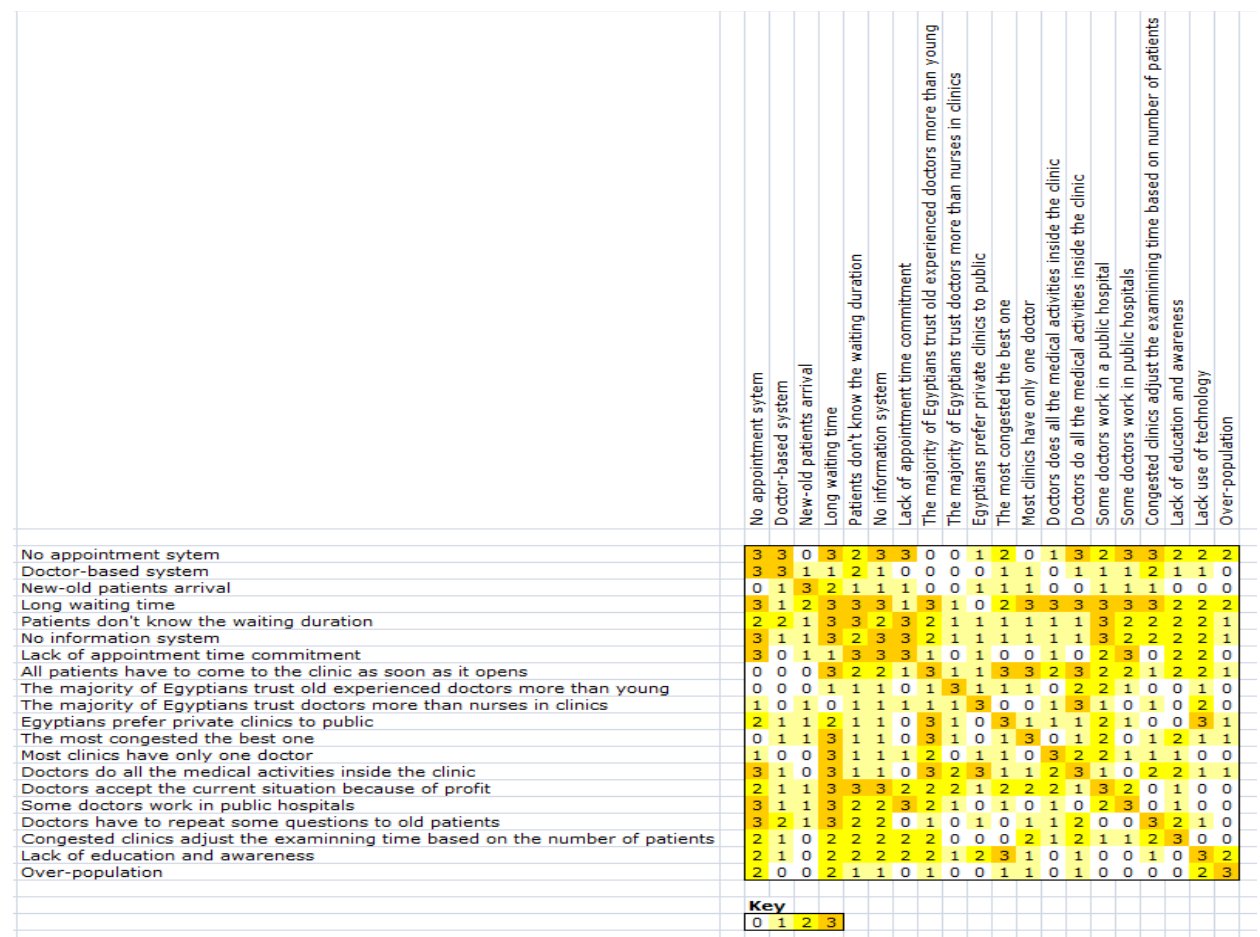

Figure 4. Problem Analysis using the Insight Matrix

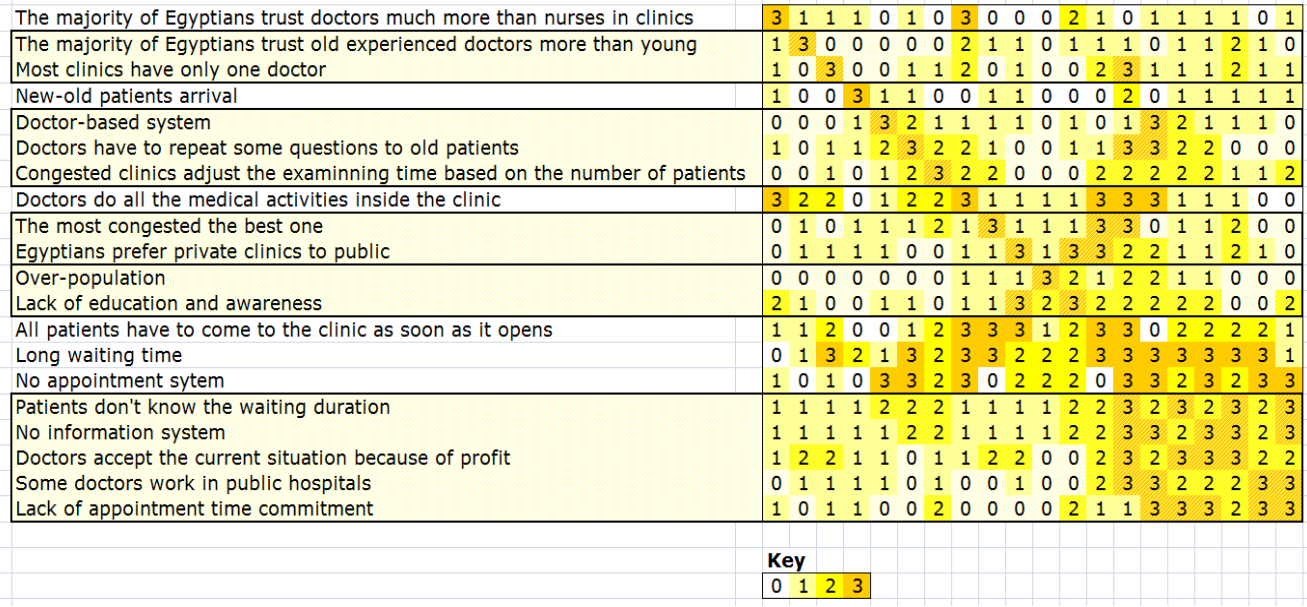

Figure 5. Snapshot of the problems clustering process

(We choose the second and the last clusters to provide a solution to them)

\section{The Application Design}

This section aims at demonstrating how the resulted information problem can be used in order to resolve the problems. Based on the design problems from the design analysis, we synthesized a concept model, information architecture, and multiple prototypes with specific features and functions for Egyptian private clinics.

\subsection{The Concept Model}

Figure 6 presents a concept model for the designed application. The application can be used through smart phones by three different users: patients, physicians, and nurses. Patients can easily search for their preferred clinics based on its available information and then select the preferred day and time from the 
available time slots. Another feature of this application is the online consultation; the patient can easily submit his/her medical complaints and an online consultation will be conducted for him/her. Physicians can easily $\log$ in to the application using their username and passwords to know their future schedule for the next week. The third user of this application is the nurse; she can follow up the schedule by coordinating with the physician, see the booking notifications and communicate with patients.

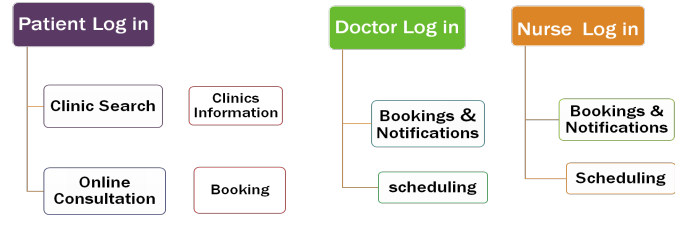

Figure 6. The concept model of the designed application

\subsection{Information Architecture}

The information architecture of the proposed application is depicted in figure 7 . The three users can make their own accounts if they are new users and can benefit from the available functions and features of the proposed application. The application name is "YourClinic" for hopefully making clinics easy to reach and deal as if they are owned by the patients. The application enables the three users to make new accounts and $\log$ in. After that, the patients can search via the application about the available clinics according to their preferences. After selecting the clinic, the patient can select his/her preferred day and also choose the time slot in that day. The physician can check the booking status and accordingly the application will automatically develop his/her schedule for going to the clinic. Furthermore, the application gives him the opportunity to give online prescriptions for the mild states which is so called on-line consultation. The third user who is the nurse can use the application for follow up the patients' notifications and reply on their inquiries. In addition, the application will develop the nurse schedule according to the patients' reservations.

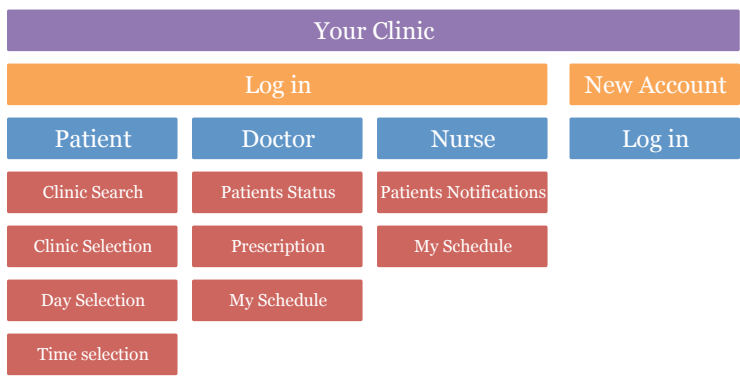

Figure 7. The information architecture of the designed application

\subsection{The application features and the developed Prototypes}

The developed prototype is an iPhone application developed using Axure software, ready for the three main users: patients, physicians, and nurses. Figure 8 is the frontage of the application in three iPhone versions, 4,5 , and 6 . The developed prototype can be retrieved from this link:

http://nlry33.axshare.com/\#c=2

The application has 6 features as follows:
1. Making Accounts
2. Clinic Search
3. Patient Booking
4. Nurse Scheduling
5. Doctor Scheduling
6. Online Consultation

These features were presented to three representatives of personas; patient, physician, and nurse and they were satisfied with these new features. We believe that the application, namely YourClinic, would increase the service quality by effectively managing the time for both service receivers and service providers.

The following are the expected impact upon patients, doctors, and nurses:

1. Less waiting time

2. Limited infection

3. Easy clinic search and booking

4. Knowing the number of patients previously

5. Better schedules

6. Less patients stress

7. Less working hours

The first three benefits are for patients, the next two benefits are for doctors, and the last two benefits are for nurses. 


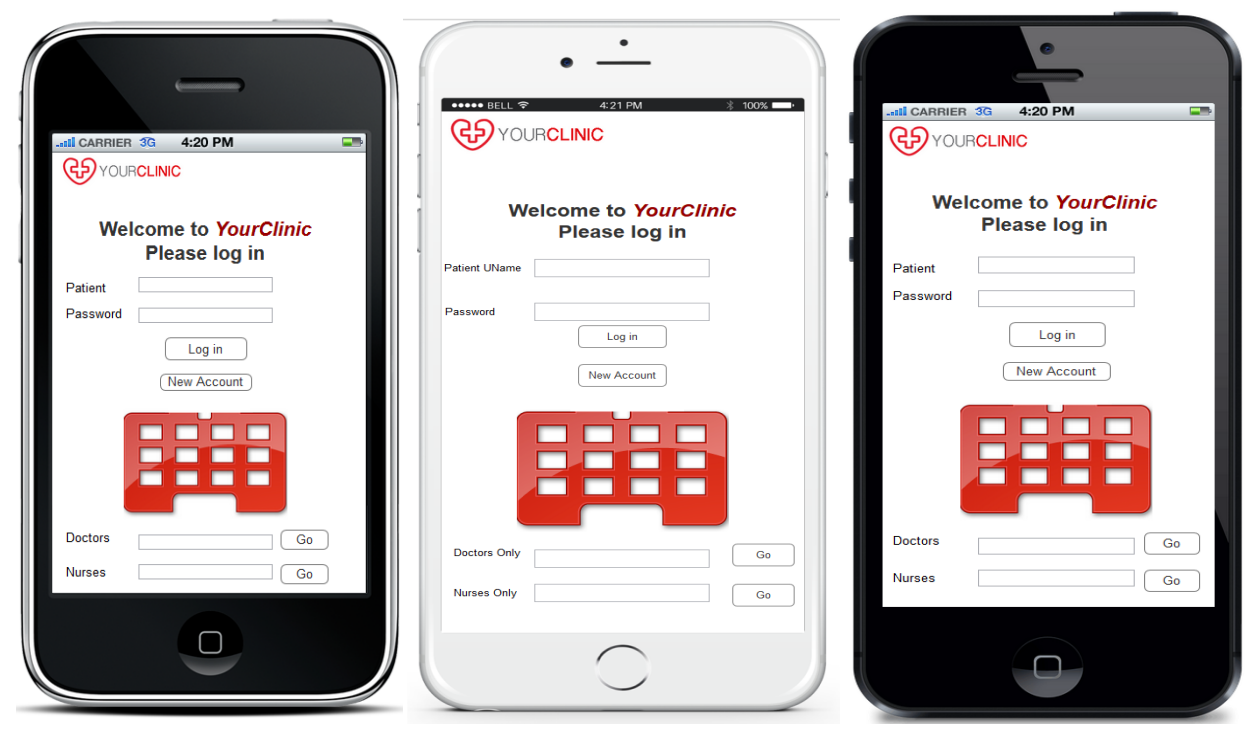

Figure 8 . The application in three iPhone versions

(To explore the prototype: http://nlry33.axshare $. \mathrm{com} / \# \mathrm{c}=2$ )

\section{Conclusions}

Traditionally, the community of healthcare service has dealt with developing new physical products. In this paper, the focus was on how make outpatient clinics more patient-oriented. To address this issue, a design thinking paradigm was invited to explore the opportunities of improving the performance in the Egyptian outpatient private clinics from the customers' perspectives.

New functions and features are developed and implemented for a smart phone application which is expected to contribute in transferring systems from physician-oriented to patient-oriented clinics and thus enhancing the service quality in the Egyptian private clinics and likewise clinics system which have similar features. By using this application, clinic staffs and patients can exploit their time effectively which leads to a sustainable healthcare system. The impact of this work would increase the effectiveness of private outpatient clinics especially in Egypt.

As a future work, a business model for this application will be developed. It is expected that this business model would contain three intersected circles consist of advertisement, competition, and payment.

\section{References}

[1] TheGuardian, "The health spending map of the world," 2012. [Online]. Available: http://www.theguardian.com/news/datablog/interacti ve/2012/jun/30/health-spending-map-world.
[2] worldbank, "Health expenditure." [Online]. Available:

http://data.worldbank.org/indicator/SH.XPD.TOTL. ZS.

[3] M. Heshmat and A. Eltawil, "Comparison between Outpatient Appointment Scheduling and Chemotherapy Outpatient Appointment Scheduling," Egypt. Int. J. Eng. Sci. Technol., vol. 19, no. 2, pp. 326-332, 2016.

[4] A. P. Vijayan, "Effect Of Appointment Schedules on The Operational Performance of a University Medical Clinic,” Louisiana State University, 2015.

[5] L. R. Laganga and S. R. Lawrence, "Appointment Scheduling with Overbooking to Mitigate Productivity Loss from No-Shows," in Conference proceedings of Decision Sciences Institute Annual Conference, 2007, pp. 1-29.

[6] B. P. Berg, B. T. Denton, B. Berg, and B. T. Denton, "Optimal booking and scheduling in outpatient procedure centers," Comput. Oper. Res., vol. 50, no. June 2015, pp. 24-37, 2014.

[7] X. Wu, M. T. Khasawneh, J. Hao, and Z. Gao, "Outpatient Scheduling in Highly Constrained Environments: A Literature Review," in The 19th International Conference on Industrial Engineering and Engineering Management, Springer, 2013, pp. 1203-1213.

[8] T. Cayirli and E. Veral, "Outpatient scheduling in health care: A review of literature," Prod. Oper. Manag., vol. 12, no. 4, pp. 519-549, 2003.

[9] D. Gupta and B. Denton, "Appointment scheduling in health care: Challenges and opportunities," IIE Trans., vol. 40, no. 9, pp. 800-819, 2008.

[10] M. A. Alkureishi et al., "Impact of Electronic Medical Record Use on the Patient - Doctor 
Relationship and Communication: A Systematic Review," Gen. Intern. Med., 2015.

[11] V. S. and A. Chaudhary, "International Journal Of," Int. J. Recent Sci. Res., vol. 7, no. 4, 2016.

[12] N. D. B. Abd, D. Munoz, and M. Ventura, "A Mixed-Methods Research Framework for Healthcare Process Improvement 1," J. Pediatr. Nurs., vol. In Press, 2015.

[13] H. B. Almarri, R. Juric, and B. Mughal, "Semantic Selection of Healthcare Apps," in 2016 49th Hawaii International Conference on System Sciences Semantic, 2016, pp. 3287-3287.

[14] S. W. Ong et al., "Article Integrating a Smartphone - Based Self - Management System into Usual Care of Advanced CKD," Clin. J. Am. Soc. Nephrol. Object., vol. 11, pp. 1-9, 2016.

[15] H. Pavliscsak et al., "Assessment of patient engagement with a mobile application among service members in transition," pp. 1-9, 2015.

[16] P. Chowdhary, S. Lee, J. Timm, H. Ludwig, and S. Knoop, "Coordinating Analytics Methods for Mobile Healthcare Applications," in 2016 International Workshop on Software Engineering in Healthcare Systems, 2016, pp. 1-4.

[17] T. Brown, P. Ehn, L. E. Associates, and P. Hillgren, "Design Things and Design Thinking: Contemporary Participatory Design Challenges Erling Bjögvinsson , Pelle Ehn , Per-Anders Hillgren," DesignIssues, vol. 28, no. 3, pp. 101-116, 2012.

[18] N. Carroll and I. Richardson, "Aligning Healthcare Innovation and Software Requirements through Design Thinking," in 2016 International Workshop on Software Engineering in Healthcare Systems, 2016, pp. 1-7.

[19] I. Green, K. Dunn, F. Bourgeois, J. Rogers, and V. W. Chiang, "Healthcare From development to implementation - A smartphone and email-based discharge follow-up program for pediatric patients after hospital discharge," Healthcare, vol. In Press, pp. 1-6, 2016.

[20] P. S. Hsueh, H. Chang, and S. Ramakrishnan, "Next Generation Wellness: A Technology Model for Personalizing Healthcare," in Healthcare Information Management Systems: Cases, Strategies, and Solutions, Health Informatics, Springer, 2016, pp. 355-374.

[21] J. B. Macdonald, "Design thinking in online communities: suggestions for overcoming crowdsourcing issues Mohammad Niamat Elahee," Int. J. Innov. Learn., vol. 19, no. 3, pp. 286-298, 2016.

[22] C. A. Watkins, G. H. Loudon, S. Gill, and J. E. Hall, "Can Design Thinking Be Used To Improve Healthcare In Lusaka Province, Zambia?," in International Design Conference - Design 2014 Dubrovnik - Croatia, May 19 - 22, 2014, 2014, pp. $1-11$.

[23] M. Cheung, "Design thinking in healthcare: Innovative Product Development through the iNPD process," Des. J., vol. 15, no. 3, pp. 299-324, 2015.

[24] D. Paula, "Understanding Design Thinking in Design Studies (2006-2015): A Systematic Mapping Study," 2016, no. May.

[25] P. G. Rowe, Design Thinking. MIT Press, 1987.

[26] K. Dorst, "The core of 'design thinking' and its application,” Des. Stud., vol. 32, no. 6, pp. 521-532, 2011.

[27] G. Ambrose and P. Harris, Design Thinking. AVA Publishing SA 2010, 2010.

[28] V. Kumar, 101 design methods: A structured approach for driving innovation in your organization. John Wiley \& Sons, 2012.

[29] A. Azab, N. Mostafa, and J. Park, "OnTimeCargo : A Smart Transportation System Development in Logistics Management by a Design Thinking Approach," in Pacific Asia Conference on Information Systems, 2016. 